\title{
Acute respiratory failure in patients with Guillain-Barré syndrome and myasthenic crisis treated with plasmapheresis in the intensive care unit
}

\author{
Wojciech Szczeklik', Miłosz Jankowski', Wojciech Węgrzyn'1, Wiesław Królikowski', Grażyna Zwolińska², \\ llona Mitka', Bożena Seczyńska', Rafał Niżankowski' \\ 1 II Department of Internal Medicine, Jagiellonian University School of Medicine, Kraków, Poland \\ ${ }^{2}$ Department of Neurology, Jagiellonian University School of Medicine, Kraków, Poland
}

\begin{abstract}
We present the cases of two patients with Guillain-Barré syndrome and one with myasthenic crisis who developed acute respiratory failure and needed mechanical ventilation in the intensive care unit. All the patients were treated with plasmapheresis, resulting in weaning from mechanical ventilation, and eventually complete functional recovery. Early treatment with plasma exchange shortens the treatment duration, whereas early intubation can prevent life-threatening complications.
\end{abstract}

Key words: acute respiratory failure, Guillain-Barré syndrome, myasthenia gravis, plasmapheresis

\section{INTRODUCTION}

Acute respiratory failure is one of the most frequent reasons for hospitalization in intensive care units (ICU) [1]. Dyspnea, reflected by hypoxemia and in some cases also by hypercapnia and respiratory acidosis in blood gas analysis, dominates in the clinical presentation. A disproportion between alveolar ventilation and pulmonary blood flow is usually the reason for hypoxemia and less often gas diffusion impairment across the air-blood barrier. The main mechanism of hypercapnia is alveolar hypoventilation which may be of neurological origin. The most frequent disorders associated with the peripheral nervous system are myasthenic crisis and acute polyneuropathies, especially acute inflammatory polyradiculoneuropathy, known as Guillain-Barré syndrome (GBS). It may lead to respiratory muscle paralysis, while in myasthenia muscle fatigue increases gradually. As a result of hypoventilation atelectasis occurs, and impairment of swallowing and cough reflexe predisposes to pneumonia which aggravates the respiratory failure. In such an event an immediate initiation of respiratory failure treatment in the ICU and the most rapid causative management are crucial [1]. Plasmapheresis (plasma exchange) is the management of choice in acute respiratory failure associated with GBS and myasthenic crisis $[2,3]$. The

\footnotetext{
Correspondence to:

Wojciech Szczeklik, MD, PhD, II Katedra Chorób Wewnętrznych, Collegium Medicum Uniwersytetu Jagiellońskiego, ul. Skawińska 8 31-066 Kraków, Poland, phone: +48-12-430-51-69, fax: +48-12-430-52-03, e-mail: wszczeklik@gmail.com Received: December 11, 2007. Accepted in final form: February 7, 2008.

Conflict of interest: none declared.

Pol Arch Med Wewn. 2008; 118 (4): 239-242

Translated by Elżbieta Cybulska, MD

Copyright by Medycyna Praktyczna, Kraków 2008
}

article presents a report on three severe cases of these diseases where mechanical ventilation and plasmapheresis treatment have been successfully used.

\section{CASE REPORTS}

\section{Individuals with Guillain-Barré syndrome}

1. A 48-year-old female patient has been transferred to the ICU of our Department from the Department of Neurology at the Jagiellonian University in Krakow. She had a history of a two-day diarrhea, nausea and fatigue which had occurred 2 weeks earlier. Within 1 week of the symptoms resolution, fasciculation, paresthesiae, fatigue and subsequently flaccid paralysis of upper extremities and a marked paresis of lower extremities developed. Based on the patient history and typical cerebro-spinal fluid findings, GBS was diagnosed. With the exacerbation of dyspnea and respiratory muscle fatigue, the patient was transferred to the ICU where she was intubated (despite the absence of significant abnormalities in the chest X-ray, arterial blood gases performed on passive oxygen therapy with $\mathrm{FiO}_{2}$ 0.5: $\mathrm{pO}_{2} 65 \mathrm{mmHg}, \mathrm{pCO}_{2}$ $50 \mathrm{mmHg}$ ) and mechanical lung ventilation was undertaken. Courses of plasmapheresis were introduced. On the $3 \mathrm{rd}$ day mechanical ventilation was weaned and intubation tube removed, in 2 weeks the patient was able to walk alone, and after one month she recovered completely. A total of 10 plasmaphereses was performed.

2. A 25-year-old male patient, not treated previously, had a mild upper respiratory tract infection three weeks before ad- 


\section{CASE REPORTS}

mission to the ICU. Several days later, tactile impairment and paresthesiae of lower and later of upper extremities occurred. He was admitted to the hospital where the cerebrospinal fluid was examined and the test demonstrated protein cell dissociation (increased protein level up to $1.8 \mathrm{~g} / \mathrm{l}$, with normal cell count). There was characteristic slowing of conduction in peripheral nerves shown in electromyography, therefore GBS was diagnosed. Despite the intravenous immunoglobin infusion, a rapid disease progression was observed which after several days led to flaccid quadriplegia (only head and neck movements were preserved) and to respiratory failure. Aspiration occurred and subsequently pneumonia developed. The patient was intubated, mechanical ventilation was initiated and he was transferred to the ICU. On admission, the patient condition was critical, because of hemodynamic instability infusion of sympathomimetic amines was required. The chest $\mathrm{X}$-rays demonstrated diffuse interstitial lung infiltrates. Arterial blood partial oxygen pressure $\left(\mathrm{PaO}_{2}\right)$ with passive oxygen therapy at $100 \%$ oxygen $\left(\mathrm{FiO}_{2} 1.0\right)$ was $70 \mathrm{mmHg}$. On neurological examination, apart from tetraplegia, there was a complete absence of deep tendon reflexes and feeling of vibration. Because of fever and presence of a resistant to meticilin coagulase-negative Staphyllococci in blood culture and Acinetobacter baumanni in bronchoalveolar lavage, vancomycin and piperacillin were administered in combination with tazobactam according to the antibiogram, with the maintenance of the earlier administered metronidazol. Plasmapheresis was immediately started with an exchange of about 3 liters plasma every 2nd day. A gradual improvement of the respiratory function was observed, and on the 6th day the mechanical ventilation was weaned. Extremities mobility improved much slower. After 6 weeks of treatment and 11 plasmaphereses the patient started to walk with assistance, was able to eat by himself and did not require further oxygen therapy; he was transferred to the Department of Neurology. A full motor efficiency recovery took over 5 months.

\section{A patient with myasthenic crisis}

A 49-year-old patient with myasthenia diagnosed 5 years ago, with documented specific acetylocholine receptor autoantibodies, on long-term corticosteroids, after thymectomy performed 2 years ago, was admitted to the ICU with myasthenic crisis. He was previously hospitalized in the Department of Neurology where head drop, ptasis, diplopia, inability to elevate the extremities and paresis intensification with effort were reported. Despite treatment with cholinesterase inhibitors (ChE), corticosteriods, and high-dose azathioprine, a sudden cardiac arrest took place in the Department of Neurology as a result of enhanced medullary symptoms and fluid aspiration. On admission to the ICU, the patient was intubated and mechanically ventilated. In order to clean the bronchi, bronchofiberoscopy was performed. Subsequently plasmaphereses, along with antibiotic therapy (empirical at first, later guided against Acinetobacter baumanni and Klebsiella pneu- moniae cultured in the bronchoalveolar lavage fluid - imipenem) were started. Considering a low hemoglobin concentration (up to $7.8 \mathrm{~g} / \mathrm{dl}$ ), red cell concentrate was transfused. Corticosteroid and ChE inhibitor therapy was continued. Lung mechanical ventilation was discontinued after a few days. After 5 plasmaphereses, the medullar symptoms and diplopia resolved and the eye bulb movements were back to normal. The extremities muscle strength demonstrated a gradual improvement. The patient was discharged to the Department of Neurology.

\section{DISCUSSION}

A total plasma exchange allows rapid elimination of autoantibodies and proinflammatory cytokines giving hope for control over exacerbated autoimmune diseases, however, positive results have been obtained only in few diseases, including GBS and myasthenic crisis [2,3]. In both diseases an obvious advantage of plasmapheresis over high doses of intravenous immunoglobulins has not been demonstrated, however, rapid effects of plasmapheresis support this therapeutic approach $[4,5]$. The efficiency of intravenous corticosteroids has not been confirmed either, and oral administration of these drugs may even slow down fitness recovery in GBS patients $[6,7]$. Making decisions regarding plasmapheresis treatment may raise fear of complications associated with the necessity of superior vena cava catheterization with a large diameter catheter, anticoagulation, removal of coagulation factors during the procedures and the risk of infection.

The plasmapheresis efficiency in the treatment of severe forms of GBS, has been demonstrated in several clinical studies. Best effects were reported, with the procedure started less than 2 weeks before the disease onset and beneficial effects even up to 4 weeks $[8,9]$. The indications are: respiratory failure, medullar paralysis and immobility. Only one single-center, open, randomized trial on the number of plasmaphereses in GBS, with intention-to-treat analysis was performed, included in the Cochrane systematic review. According to the study among patients not requiring mechanical ventilation, 4 procedures were more efficient than 2 , and in mechanically ventilated patients increasing the number of plasmaphereses to 6 did not improve the treatment results compared to 4 procedures [4]. It seems, however, that the decision on the number of plasmaphereses should be individualized depending on their clinical condition.

Plasmapheresis introduced in the early stage of GBS, an early intubation and mechanical ventilation allow to avoid the most severe complications: aspiration pneumonia and severe respiratory failure [10-12], a good example of which are the patients presented in this study. Plasmapheresis was introduced in the young man already after bronchial aspiration and pneumonia development. The acute respiratory distress syndrome (ARDS) diagnostic criteria were already fulfilled. Bilateral interstitial lung lesions and the oxygenation 
index; the ratio $\mathrm{PaO}_{2}$ and fraction of inspired oxygen $\left(\mathrm{FiO}_{2}\right)$ $<200 \mathrm{mmHg}$ (precisely $70 \mathrm{mmHg} ; \mathrm{PaO}_{2}=70 \mathrm{mmHg}, \mathrm{FiO}_{2}$ $=1.0$ ) were observed. The mobility recovery took much longer in comparison with the above presented female patient. Plasmaphereses and mechanical ventilation was introduced nearly immediately after respiratory failure symptoms findings; respiratory muscles exhaustion, which enabled to avoid pulmonary (pneumonia, ARDS) and systemic (sepsis) complications, which had occurred in the GBS male patient and allowed quicker recovery.

The patient with myasthenic crisis is an another example, confirming that delay in mechanical ventilation introduction with already present respiratory muscles exhaustion can lead to serious complications. Plasmaphereses are also the management of choice in myasthenic crisis, especially when the earlier attempts of treating with $\mathrm{ChE}$ inhibitors, corticosteroids and immunosuppressive drugs were ineffective. The therapeutic plasmapheresis effect is mainly due to the elimination of circulating acetylocholine receptor antibodies elevated in over $90 \%$ of patients with generalized myasthenia. Another important aspect of such treatment is the elimination of factors able to cause the crisis, such as respiratory tract infection [8], as it probably occurred in the presented case.

In the presented patients there were no serious plasmaphereses complications since the principles of aseptic techniques during vessel catheter introduction, care and procedure performance were strictly followed, as well as coagulation system was monitored. Anaemia (without enhanced hemolysis) occurred in the male patient with myasthenia, which required a single transfusion of red cells. In that patient and in the male patient with GBS, however, a respiratory tract infection (possibly hospital-aquired) had developed before start of plasma exchange.

A careful clinical observation allows finding respiratory muscles fatigue symptoms prior to the occurrence of serious arterial blood gases abnormalities and plays a key role in decision making associated with initiation of plasmapheresis and mechanical ventilation both in myasthenic crisis and GBS patients. With marked dyspnea and rapid shallow breathing, it is not advised to delay plasmaphereses introduction until hypoxemia and hypercapnia occur; an early introduction may prevent respiratory failure aggravation which requires mechanical ventilation commencement. Spirometry happens to be very helpful in monitoring such patients; usually limited to vital capacity assessment attempt, as significant muscle weakening makes the forced expiration maneuver impossible.

In GBS and myasthenic crisis associated respiratory failure, an invasive mechanical ventilation is conducted (intubation necessary) as noninvasive methods usually support ventilation, which means they require the patient's own respiratory effort. Moreover, they do not protect the airways from aspiration and should not be employed in patients incapable of an efficient cough.

Acute respiratory failure, including its most severe form, the ARDS, has a stormy course and requires an immediate treatment. It is often caused by ventilation disorders in the course of peripheral nervous system diseases, the most frequent one being GBS and myasthenic crisis. In both cases, therapeutic plasmapheresis (plasma exchange) is the management of choice, and should be started promptly to avoid adverse events.

\section{ACKNOWLEDGMENTS}

The authors wish to thank all members of nursing personnel of the ICU, especially Mr. Ryszard Ślęzak, for his help in plasmapheresis performance and Mrs. Ewa Kurleto-Kalitows$\mathrm{ka}$, for her invaluable support.

\section{REFERENCES}

1. Tuleja E, Królikowski W, Twardowska M, et al. Acute respiratory distress syndrome (ARDS). Pol Arch Med Wewn. 2000; 103: 319-327.

2. Szczepiorkowski ZM, Bandarenko N, Kim HC, et al. Guidelines on the use of therapeutic apheresis in clinical practice: evidence-based approach from the Apheresis Applications Committee of the American Society for Apheresis. J Clin Apher. 2007; 22: 106-175.

3. Natarajan N, Weinstein R. Therapeutic apheresis in neurology critical care. J Intensive Care Med. 2005; 20: 212-224.

4. Raphaël JC, Chevret S, Hughes RAC, Annane D. Plasma exchange for Guillain-Barré syndrome. Cochrane Database Syst Rev. 2002; 2: CD001798.

5. Gajdos $P$, Chevret $S$, Toyka K. Plasma exchange for myasthenia gravis. Cochrane Database Syst Rev. 2002; 4: CD002275.

6. Hughes RAC, Swan AV, van Koningsveld R, van Doorn PA. Corticosteroids for Guillain-Barré syndrome. Cochrane Database Syst Rev. 2006; 2: CD001446.

7. Schneider-Gold C, Gajdos P, Toyka KV, Hohlfeld RR. Corticosteroids for myasthenia gravis. Cochrane Database Syst Rev. 2005; 2: CD002828.

8. Dhar R, Stitt L, Hahn AF. The morbidity and outcome of patients with Guillain-Barré syndrome admitted to the intensive care unit. J Neurol Sci. 2008; 264: 121-128.

9. McGillicuddy DC, Walker 0 , Shapiro NI, et al. Guillain-Barré syndrome in the emergency department. Ann Emerg Med. 2006; 47: 390-393.

10. Sharstar T, Chevret $S$, Bourdain $F$, et al. Early predictors of mechanical ventilation in Guillain-Barré syndrome. Crit Care Med. 2003; 31: 278-283.

11. Ali MI, Fernandez-Perez ER, Pendem S, et al. Mechanical ventilation in patients with Guillain-Barré syndrome. Respir Care. 2006; 51: 1403-1407.

12. Jani-Acsadi A, Lisak RP. Myasthenic crisis: guidelines for prevention and treatment. J Neurol Sci. 2007; 261: 127-133. 